\title{
Etude des performances génétiques des descendances parentales issues de deux systèmes de reproduction de géniteurs utilisés en production de semences sélectionnées de palmier à huile (Elaeis guineensis Jacq.)
}

\author{
Ekra Kouamé TANO ${ }^{1 *}$, Jean-Noël KONAN ${ }^{2}$, Oulo ALLA N'NAN ${ }^{1}$, René AKANVOU ${ }^{3}$, \\ Assanvo Simon-Pierre NGUETTA ${ }^{1}$ et Eugène Kouakou KONAN ${ }^{2-3}$ \\ ${ }^{1}$ Université Felix Houphouët-Boigny, UFR Biosciences, Laboratoire de génétique, 22 BP 582 Abidjan 22, \\ Côte d'Ivoire. \\ ${ }^{2}$ Centre National de Recherche Agronomique (CNRA), Station de Recherché de La Mé, 13 BP 989 ABIDJAN \\ 13, Côte d'Ivoire. \\ ${ }^{3}$ Centre National de Recherche Agronomique (CNRA), Direction Générale, 01 BP 1740 Abidjan 01, \\ Côte d'Ivoire. \\ ${ }^{4}$ Centre National de Recherche Agronomique (CNRA), Direction Régionale Abidjan, 08 BP 33 Abidjan 08, \\ Côte d'Ivoire. \\ *Auteur correspondant ; E-mail: tanoekrakouam@gmail.com, Tel: (+225) 57428704.
}

\section{RESUME}

La production de semences sélectionnées de palmier à huile passe nécessairement par l'obtention de géniteurs parentaux sélectionnés à l'issue des tests d'hybrides. Les semences de deuxième cycle de sélection récurrente en diffusion proviennent de deux groupes de géniteurs parentaux ayant la même origine, mais structurés différemment. Le premier groupe est constitué de géniteurs élites de premier cycle ayant subi deux générations successives d'autofécondation de type $[(\mathrm{G} 1)$ AF] AF. Le deuxième groupe est composé de géniteurs ayant subi un cycle préalable de recombinaison entre géniteurs de type (G1 x G2) AF. Cette étude a consisté à comparer les performances génétiques des géniteurs issues des autofécondations parentales. Elle a porté sur 1189 géniteurs Dura issus des champs semenciers des catégories C1001F, C2501 et C7001. Les mesures ont été faites sur les caractères les plus héritables comme le nombre et le poids moyen des régimes et le nombre de graines sèches sur régime. Il ressort de cette étude que la catégorie C7001 a fourni de gros régimes, avec un nombre élevé de graines sèches sur régime. La catégorie $\mathrm{C} 1001 \mathrm{~F}$ a enregistré un petit nombre de petit régime avec peu de graines sèches sur régime. La catégorie C2501 a donné un grand nombre de petit régime. La comparaison des performances des deux systèmes de reproduction de géniteurs a révélé que les géniteurs issus de la recombinaison suivie d'une autofécondation ont donné les meilleures performances agronomiques (NR, PMR et GRS/R) par rapport à ceux dérivant de la double autofécondation. Les résultats obtenus mettent en évidence les effets défavorables de la consanguinité due au cycle successif d'autofécondation sur la performance des géniteurs.

(C) 2019 International Formulae Group. All rights reserved

Mots clés: Palmier à huile, semences, sélection récurrente réciproque, consanguinité.

\section{Study of the genetic performance of parental progeny from two spawning reproduction systems used in production of oil palm (Elaeis guineensis Jacq.) selected seeds}

\section{ABSTRACT}

The production of selected seeds of oil palm necessarily by obtaining parental spawners selected after hybrid tests. The seeds of the second cycle of recurrent diffusion selection come from two parental spawing groups having the same origin but structured differently. The first group consists of elite spawners 
undergraduate who have undergone two successive generations of self-fertilization of type [(G1) AF] AF. The second group is composed of parents who have undergone a prior cycle of recombination between spawners of type (G1 x G2) AF. The aim of this study was to compare the genetic performance of spawners from parental self-fertilization. To this end, 1189 parents of Dura from the seed fields of categories C1001F, C2501 and C7001 were used. Measurements have been made on the most heritable traits like number of bunches, mean bunch weights, and number of dry seeds on bunch. The C7001 category produced a big bunch with a high number of dry seeds on a bunch. The $\mathrm{C} 1001 \mathrm{~F}$ category produced a little bunch with few dry seeds on bunch. The C2501 category gave a lot of small bunch. Number of bunches, mean bunch weigh and number of dry seeds on bunch were lower in double-self-fertilization broodstocks than in broodstock. Recombination followed by self-fertilization. The results obtained highlight the adverse effects of consanguinity due to the successive cycle of selfing on the performance of the parents.

(C) 2019 International Formulae Group. All rights reserved

Keywords: Oil palm, seeds, reciprocal recurrent selection, consanguinity.

\section{INTRODUCTION}

Parmi les plantes oléagineuses, le palmier à huile (Elaeis guineensis, Jacq.) est celle qui a le plus gros rendement d'huile par hectare, avec en moyenne 4 tonnes/ha/an (Rival et Levang, 2014; Corley et Tinker, 2016). Dans les conditions agropédoclimatiques favorables, ce rendement en huile peut atteindre 7 à 9 tonnes/ha/an (Durand-Gasselin et al., 2011). Actuellement, la production mondiale d'huile de palme est estimée à environ 62 millions de tonne (USDA 2017), dépassant largement l'huile de soja qui était jusqu'en 2005, la première source mondiale d'huile végétale (Cheng, 2010).

En Côte d'Ivoire, le verger de palmier à huile couvre environ 350000 hectares, avec une production nationale annuelle d'huile de palme brute d'environ 400000 tonnes dont $55 \%$ sont exportés. Le palmier à huile est la $2^{\mathrm{e}}$ culture d'exportation après le cacao (1 300000 tonnes) (Osseni, 2009). Cet accroissement de la production est dû à une augmentation des surfaces de plantation mais aussi à la qualité du matériel planté.

Le matériel végétal utilisé est constitué d'hybrides obtenus à partir d'un schéma de sélection récurrente réciproque (Meunier et Gascon, 1972) qui permet le choix des meilleurs croisements. La diffusion aux planteurs des individus issus de ces croisements se fait par voie de semences. Plusieurs cycles d'autofécondation ont été réalisés et permettent aujourd'hui de diffuser les semences de $2^{\text {nd }}$ cycle. Deux stratégies de reproduction de ces géniteurs élites ont été adoptées pour la production des semences améliorées de $2^{\text {nd }}$ cycle (Gascon et al., 1981). Il s'agit de la voie utilisant deux cycles successifs d'autofécondation de type AFAF et la voie basée sur un cycle de recombinaison suivie d'un cycle d'autofécondation de type AFSIB entre géniteurs élites apparentés (plein frères, demi-frères, etc.). Les semences produites par ces deux méthodes ont toujours été diffusées en mélange. Tano et al. (2017) ont montré au cours de leurs travaux que les géniteurs de type AFSIB issus de recombinaison suivie d'une autofécondation ont une vitesse de croissance plus élevée que ceux de type AFAF obtenus à partir de deux cycles successifs d'autofécondation. L'hypothèse selon laquelle des différences de performances agronomiques pourraient exister entre les populations de ces géniteurs issus de ces deux systèmes de reproduction se pose.

La présente étude vise à faire une comparaison entre les géniteurs de ces deux stratégies de reproduction de géniteurs utilisés en production de semences à travers une caractérisation agronomique (composantes de la production de régimes) afin d'opter pour la meilleure stratégie de création des futurs champs semenciers.

\section{MATERIEL ET METHODES Matériel végétal}

Le matériel végétal est composé de mille cent quatre-vingt-neuf (1189) géniteurs de type Dura utilisés dans les champs semenciers de second cycle de sélection récurrente réciproque du palmier à huile en Côte d'Ivoire. Ces géniteurs ont été obtenus, 
soit par une double autofécondation (AFAF), soit par une recombinaison suivie d'une autofécondation entre palmiers apparentés (AFSIB) (Tableau 1).

En fonction de l'origine des parents croisés, ces géniteurs ont été regroupés en trois ensembles ou catégories qui sont C2501, $\mathrm{C} 1001 \mathrm{~F}$ et $\mathrm{C} 7001$ dont les géniteurs sont issus des croisements DA115D x DA3D, DA115D x DA115D et DA5D x DA3D, respectivement. A l'intérieur de chaque catégorie, les descendances de type AFAF et de type AFSIB ont été évaluées. Les palmiers utilisés sont âgés de 15 à 21 ans.

\section{Dispositif expérimental}

Les champs semenciers d'où proviennent les géniteurs évalués ont été mis en place entre 1994 et 2002 à la station de recherche de La Mé, Côte d'Ivoire. Ils ont été plantés suivant un dispositif en lignées repérées, à une densité de 143 arbres par hectare.

\section{Variables mesurées}

Les observations ont été réalisées de 2008 à 2011 sur les variables se rapportant à la composante de la production de régimes:

\section{- Le nombre (NR) et le poids moyen de régime (PMR)}

Le nombre de régimes (NR) et le poids total des régimes (PR) sont enregistrés à chaque récolte à l'arrivée des régimes dans l'unité de préparation des semences. Le nombre de régimes (NR) et le poids total des régimes $(\mathrm{PR})$ ont été enregistrés arbre par arbre et ont permis de déterminer le poids moyen des régimes (PMR) selon la formule :

$\mathrm{PMR}=\frac{\mathrm{PR}}{\mathrm{NR}}$

$P M R=$ Poids Moyen de Régime, $P R=$ Poids total de Régime, $N R=$ Nombre de Régime

- Le nombre de graines sèches sur régimes (NGSR)

Le nombre moyen de graines sèches sur régime est déterminé par le rapport entre le nombre total de graines obtenues sur le nombre moyen de régimes récoltés pendant les quatre années d'étude sur chaque géniteur étudié.

NGSR $=\frac{\text { TGS }}{\text { NTR }}$
NGSR = Nombre de Graines Sèches sur Régime, $T G S=$ Total de Graines Sèches, NTR = Nombre Total de Régime par géniteur

Chaque régime mâture récolté subit, après la pesée, un égrappage qui consiste à séparer les épillets du rachis à l'aide d'une hachette dans un casier grillagé. Les épillets sont humidifiés et stockés pendant 3 à 4 jours pour faciliter leur effruitage. Les fruits sont ensuite mis dans un bac de fermentation pendant trois jours également. $\mathrm{La}$ fermentation est accompagnée d'un arrosage régulier. Les fruits fermentés sont dépulpés mécaniquement (Bernard et Malingraux, 1965). A la sortie du dépulpage, les graines sont placées dans des caissettes en bois dans une salle climatisée pour le ressuyage et le séchage. Une finition au couteau est souvent nécessaire après le ressuyage pour enlever les fibres qui restent autour de la noix. A la suite de l'essuyage, les graines blanches et les graines anormalement petites sont éliminées par triage. Les graines sont enfin comptées par régime et stockées pendant 3 à 6 mois au magasin de stockage.

\section{Analyses statistiques}

Des analyses descriptives (calculs de moyennes, écart-types, coefficients de variation, intervalles de confiance) ont été réalisées pour décrire les différentes descendances évaluées.

Le test de Levene a été utilisé pour vérifier les hypothèses de normalité et d'homogénéité des variances des variables, nécessaires à l'utilisation des tests paramétriques. Ensuite, une analyse de variance (ANOVA) à un facteur (Bouroche et Saporta, 1980) suivie du test de comparaison multiple des moyennes de Newman-Keuls au risque de 5\% (Dagnelie, 2012) a été appliquée pour effectuer la comparaison des moyennes des variables mesurées des trois catégories de semences

L'effet du système du système de la reproduction a été évalué à l'aide du test $t$ de Student a afin de vérifier si le mode de reproduction des géniteurs de champs semenciers impactait les composantes de production de régimes. Toutes ces analyses ont été effectuées à l'aide du logiciel Statistica 7.1 . 
Tableau 1 : Matériel végétal étudié.

\begin{tabular}{|c|c|c|c|c|c|}
\hline Catégorie & $\begin{array}{l}\text { Origine des } \\
\text { différentes catégories }\end{array}$ & $\begin{array}{l}\text { Croisements entre les descendants de } \\
\text { la première génération des catégories }\end{array}$ & $\begin{array}{l}\text { Système de } \\
\text { reproduction }\end{array}$ & $\begin{array}{c}\text { Descendances issues de la deuxième } \\
\text { génération des catégories }\end{array}$ & $\begin{array}{l}\text { Nombre de géniteurs Dura } \\
\text { évalués, issus des descendances } \\
\text { de la deuxième génération }\end{array}$ \\
\hline \multirow{6}{*}{ C7001 } & \multirow{6}{*}{ DA115D x DA3D } & (LM5357D X LM5373D) AF & AFSIB & LM19955 & 96 \\
\hline & & (LM5373D) AFAF & AFAF & LM19759 & 30 \\
\hline & & (LM5373D X LM5570D) AF & AFSIB & LM20983 & 107 \\
\hline & & (LM5373D) AFAF & AFAF & LM19759 & 30 \\
\hline & & (LM5570D X LM5644D) AF & AFSIB & LM19947 & 47 \\
\hline & & (LM5644 D) AFAF & AFAF & LM19640 & 40 \\
\hline \multirow{6}{*}{$\mathrm{C} 2501$} & \multirow{6}{*}{ DA3D x DA5D } & (LM3047D X LM3604D) AF & AFSIB & LM19614 & 24 \\
\hline & & (LM3047D) AFAF & AFAF & LM19622 & 48 \\
\hline & & (LM3257D X LM3053D) AF & AFSIB & LM21189 & 25 \\
\hline & & (LM3053D) AFAF & AFAF & LM20258 & 92 \\
\hline & & (LM3043D X LM3038D) AF & AFSIB & LM19820 & 54 \\
\hline & & (LM3038D) AFAF & AFAF & LM19892 & 43 \\
\hline \multirow{8}{*}{$\mathrm{C} 1001 \mathrm{~F}$} & \multirow{8}{*}{ DA115D AF } & (LM2509D) AFAF & AFAF & LM19175 & 71 \\
\hline & & (LM2509D X LM2523D) AF & AFSIB & LM18503 & 22 \\
\hline & & (LM3394D) AFAF & AFAF & LM17114 & 61 \\
\hline & & (LM3394D X LM2519D) AF & AFSIB & LM18783 & 66 \\
\hline & & (LM2531D) AFAF & AFAF & LM19016 & 69 \\
\hline & & (LM2515D X LM2531D) AF & AFSIB & LM18805 & 100 \\
\hline & & (LM3005D) AFAF & AFAF & LM19121 & 79 \\
\hline & & (LM3394D x LM3005D) AF & AFSIB & LM18801 & 85 \\
\hline & Total & 20 & 2 & 20 & 1189 \\
\hline
\end{tabular}

AFAF : Double autofécondation, AFSIB : Autofécondation suivie de recombinaison. 


\section{RESULTATS}

\section{Composantes de la production de régimes}

Des différences significatives existent entre les catégories de géniteurs pour le nombre de régimes produits par arbre $(\mathrm{P}=$ 0,001). Les géniteurs de la catégorie C2501 ont produit le plus grand nombre de régime (4,00 régimes), suivi des géniteurs de la catégorie C7001 avec 3,60 régimes et des géniteurs de la catégorie $\mathrm{C} 1001 \mathrm{~F}$ qui ont donné 3,32 régimes (Figure 1). Une forte variabilité a été observée entre les géniteurs au sein des trois catégories. Les coefficients de variabilité ont été de $53,60 \%$ pour la catégorie $\mathrm{C} 1001 \mathrm{~F}, 54 \%$ pour la catégorie $\mathrm{C} 2501$ et $61,94 \%$ pour la catégorie C7001 (Tableau 2).

Pour ce qui concerne le poids moyen des régimes, les géniteurs de la catégorie C7001 ont produit des régimes relativement plus gros avec 19,67 kg que les géniteurs des deux autres catégories. Le poids moyen de régimes enregistré pour les géniteurs de la catégorie $\mathrm{C} 1001 \mathrm{~F}$ a été de $18,00 \mathrm{~kg}$ et de $16,65 \mathrm{~kg}$ pour les géniteurs de la catégorie C2501 (Figure 2). Comme pour le nombre de régime, la variabilité a été également élevée dans les trois catégories C1001F, C7001 et C2501 avec des coefficients de variation respectifs de $28,33 \%, 24,40 \%$ et $29,46 \%$ (Tableau 2).

\section{Nombre de graines sèches}

Le nombre de graines sèches sur régime des géniteurs a été significativement plus élevé $(\mathrm{P}=0,001)$ chez les géniteurs de la catégorie C7001 avec 950,21 GSR, suivi de la catégorie C2501 qui a enregistré 924,19 GSR. La catégorie $\mathrm{C} 1001 \mathrm{~F}$ a donné le plus faible nombre de graines sèches sur régime avec 891,20 GSR (Figure 3). La variabilité a été aussi forte au sein des trois catégories C1001F, C7001 et C2501 avec des coefficients de variation respectifs de $29,52 \%$, $35,65 \%$ et $37,02 \%$ (Tableau 2).
Effet du système de reproduction de géniteurs sur les caractéristiques agronomiques des descendances Nombre de Régime (NR)

Des différences existent au niveau des géniteurs issus de 7 couples sur les 10 évalués (70\%) $(\mathrm{P}=0,001)$, entre les deux systèmes de reproduction, à l'exception du couple 3 chez la catégorie $\mathrm{C} 1001 \mathrm{~F}$, du couple 2 chez la catégorie $\mathrm{C} 2501$ et du couple 2 de la catégorie C7001 où aucune différence n'a été obtenue (Tableau 3). Dans chaque catégorie de géniteurs, les descendances de type AFSIB issues d'autofécondation suivie de recombinaison ont donné les régimes en nombre plus élevés que ceux dérivant du système de reproduction de type AFAF à l'exception du couple 1 chez la catégorie C2501 où la descendance issue LM19622 obtenue par reproduction de type AFAF a enregistré la plus grande valeur que la descendance LM19614 dérivant de reproduction de type AFSIB.

Globalement, chez les catégories C1001F et C7001, les géniteurs issus du système de reproduction de type AFSIB ont donné un grand nombre de régime avec un faible coefficient de variation par rapport à ceux dérivant de reproduction de type AFAF qui ont donné un petit nombre de régime avec un coefficient de variation élevé. Chez la catégorie $\mathrm{C} 1001 \mathrm{~F}$, les géniteurs issus de type AFSIB ont donné en moyenne 3,9 régimes avec un coefficient de variation égal à 50,83\% contre 3,00 régimes pour les géniteurs dérivant de type AFAF avec un coefficient de variation de 56,00\%. Chez la catégorie C7001, les géniteurs issus de reproduction de type AFSIB ont fourni 3,75 régimes pour un coefficient de variation de 58,58\% contre 2,86 régimes pour les géniteurs de type $\mathrm{AFAF}$ avec un coefficient de variation de $63,74 \%$. En revanche, au niveau de la catégorie $\mathrm{C} 2501$, le nombre de régime produit n'a pas permis de différencier les deux systèmes de reproduction, cependant les géniteurs issus du système de reproduction de type AFAF ont donné un coefficient de variation de $54,86 \%$, 
plus élevé que ceux issus du système de type AFSIB avec $49,03 \%$.

\section{Poids moyen de régime (PMR)}

Des différences existent pour le poids moyen de régime entre les descendances issues des deux systèmes de reproduction. Les géniteurs issus de reproduction de type AFSIB ont donné des régimes de poids moyen significativement plus gros $(\mathrm{P}=0,001)$ que ceux issus de reproduction de type AFAF à l'exception du couple 3 chez la catégorie C7001 où aucune différence n'a été enregistrée (Tableau 4).

Globalement, les géniteurs des catégories C1001F, C2501 et C7001, descendants de reproduction de type AFSIB ont donné des régimes de poids moyens significativement plus gros avec les plus faibles coefficients de variation que ceux obtenus à partir de reproduction de type $\mathrm{AFAF}$ qui ont donné de petits régimes. Au niveau de la catégorie C1001F, les géniteurs de type AFSIB ont donné un poids moyen de $20,03 \mathrm{~kg}$ avec un coefficient de variation de $18,74 \%$ contre $15,92 \mathrm{~kg}$ pour les géniteurs de type AFAF avec un coefficient de variation de 23,08\%. Chez la catégorie C2501, les géniteurs issus de reproduction de type AFSIB ont donné un poids moyen de $18,42 \mathrm{~kg}$ avec un coefficient de variation de $22,76 \%$ contre $14,21 \mathrm{~kg}$ pour les géniteurs dérivant de type AFAF avec un coefficient de variation de $26,41 \%$. Enfin, concernant la catégorie C7001, les géniteurs de type AFSIB ont enregistré un poids moyen de régime de 20,99 $\mathrm{kg}$ et un coefficient de variation de $24,54 \%$ contre $18,89 \mathrm{~kg}$ pour les géniteurs issus de reproduction de type AFAF avec un coefficient de variation de $26,83 \%$.

\section{Nombre de graines sèches sur régime}

Des différences significatives $(\mathrm{P}=$ 0,001) ont été enregistrées entre le nombre de graines sèches sur régime fourni par les descendances issues des deux systèmes de reproduction. Les descendances issues de reproduction de géniteurs de type AFSIB ont enregistré au niveau de tous les couples chez les trois catégories, des valeurs significativement plus élevées que chez les descendances issues de reproduction de géniteurs de type AFAF (Tableau 5).

En somme, les géniteurs des catégories C1001F, C2501 et C7001, descendants des AFSIB ont donné le plus grand nombre de graines sèches sur régime et des faibles coefficients de variations par rapport à ceux dérivant de reproduction de type AFAF qui ont donné un nombre réduit de graines sèches sur régimes et des coefficients de variation les plus élevés. Au niveau de la catégorie $\mathrm{C} 1001 \mathrm{~F}$, les géniteurs issus de reproduction de type AFSIB ont donné 984,43 graines sèches sur régime avec un coefficient de variation de $22,48 \%$ contre 777,31 graines sèches sur régime pour les géniteurs dérivant de reproduction de type AFAF pour un coefficient de variation de $30,62 \%$. Chez la catégorie C2501, les géniteurs issus de reproduction de type AFSIB ont fourni 985,54 graines sèches sur régime avec un coefficient de variation de $29,66 \%$ contre 819,80 graines sèches sur régime pour les géniteurs de issus de reproduction de type AFAF pour un coefficient de variation de $34,21 \%$. Enfin, concernant la catégorie C7001, les géniteurs de type AFSIB ont enregistré 1038,60 graines sèches sur régime pour un coefficient de variation $30,91 \%$ contre 990,14 graines sèches sur régime pour les géniteurs issus de reproduction de type AFAF avec un coefficient de variation de $33,90 \%$.

\section{Comparaison des composantes de production de régimes des deux systèmes de reproduction de géniteurs}

Des différences significatives $(\mathrm{P}=$ 0,001) ont été observées entre les composantes de production de régimes des deux systèmes de reproduction de l'ensemble des géniteurs. Les descendants obtenus par recombinaison de type AFSIB ont généralement enregistré les meilleures performances agronomiques par rapport aux descendants issus d'autofécondation de type AFAF. 
En revanche, les variabilités ont été plus importantes chez les géniteurs issus de reproduction de type AFAF qu'au niveau des géniteurs issus de reproduction de type AFSIB. Au niveau du nombre de régime fourni, les géniteurs obtenus à partir de la reproduction de type AFSIB ont donné 4,07 régimes avec 48,64\% de coefficient de variation contre 3,95 régimes avec un coefficient de variation de $56,72 \%$ pour les géniteurs obtenus à partir de la reproduction de type AFAF. Concernant le poids moyen de régime, les géniteurs de type AFSIB ont enregistré des régimes de $19,31 \mathrm{~kg}$ contre
$16,24 \mathrm{~kg}$ pour les régimes dérivant de des géniteurs de type AFAF. La variabilité a été de 25,81\% chez les géniteurs de type AFSIB contre $27,77 \%$ dans les systèmes de reproductions de type AFAF. Enfin, pour le nombre de graines sèches sur régime, les géniteurs obtenus à partir de reproduction de type AFSIB ont fourni 980,92 GSR contre 831,35 GSR pour les géniteurs obtenus à partir de reproduction de type AFAF. La variabilité enregistrée a été de $30,87 \%$ chez les géniteurs provenant de reproduction de type AFSIB contre $35,34 \%$ au niveau des géniteurs issus de type AFAF (Tableau 6).

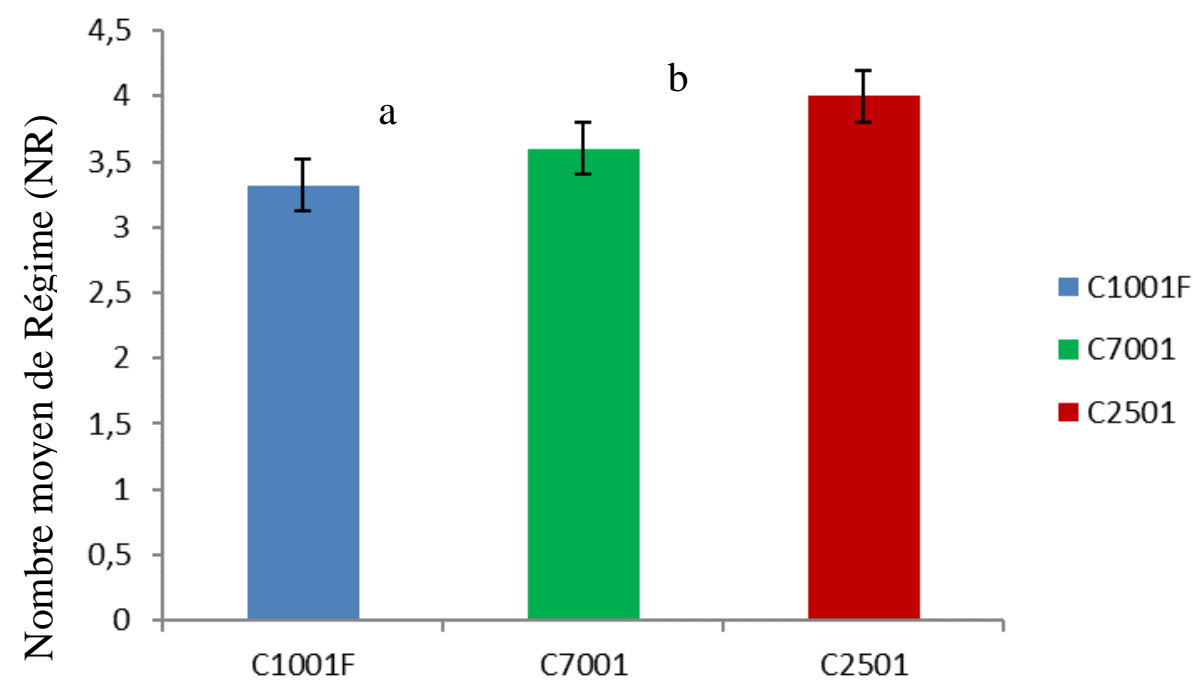

Catégories de géniteurs

Les graphiques suivis de la même lettre ne sont pas statistiquement différents au seuil de $\alpha=0,05$ par le test de KnewmanKeuls.

Figure 1: Comparaison du nombre moyen de régime des trois catégories de géniteurs Dura.

Tableau 2 : Variabilité des composantes de la production de régimes des catégories de géniteurs.

\begin{tabular}{lcccccc}
\hline Catégories & NR & Cv $(\%)$ & PMR $(\mathbf{k g})$ & Cv $(\%)$ & NGSR & Cv (\%) \\
\hline C1001F & $3,32 \pm 0,08^{\mathrm{a}}$ & 53,60 & $18,00 \pm 0,24^{\mathrm{a}}$ & 28,33 & $891,20 \pm 10,16^{\mathrm{a}}$ & 29,52 \\
C7001 & $3,60 \pm 0,13^{\mathrm{b}}$ & 54,00 & $19,67 \pm 0,29^{\mathrm{b}}$ & 24,40 & $950,21 \pm 22,15^{\mathrm{b}}$ & 35,65 \\
C2501 & $4,00 \pm 0,12^{\mathrm{c}}$ & 61,94 & $16,65 \pm 0,28^{\mathrm{c}}$ & 29,46 & $924,19 \pm 21,33^{\mathrm{c}}$ & 37,02 \\
\hline
\end{tabular}

$\mathrm{NR}$ : Nombre de régimes ; PMR : Poids moyen du régime $(\mathrm{kg})$; NGSR : Nombre de graines sèches sur régime, $\mathrm{Cv}$ : Coefficient de variation. 


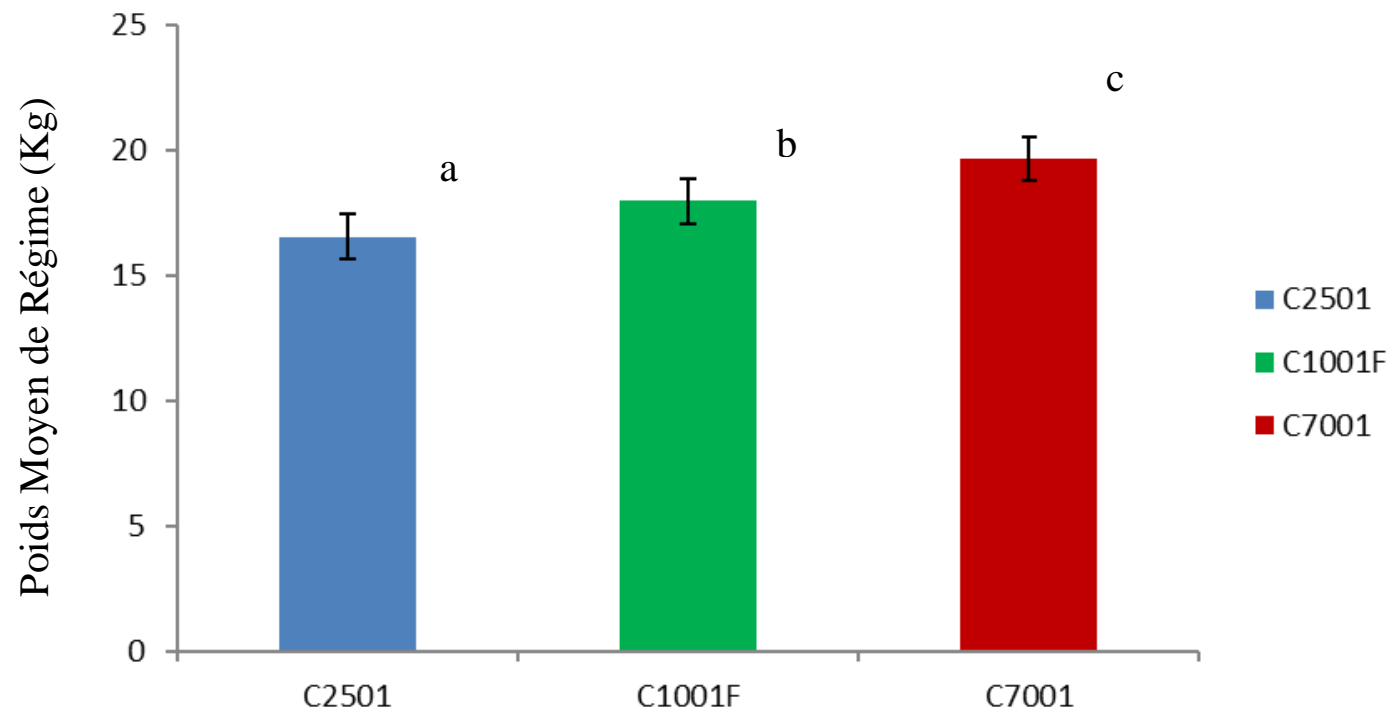

Catégories de géniteurs

Les graphiques suivis de la même lettre ne sont pas statistiquement différents au seuil de $\alpha=0,05$ par le test de KnewmanKeuls.

Figure 2 : Comparaison du poids moyen de régime des trois catégories de géniteurs Dura.

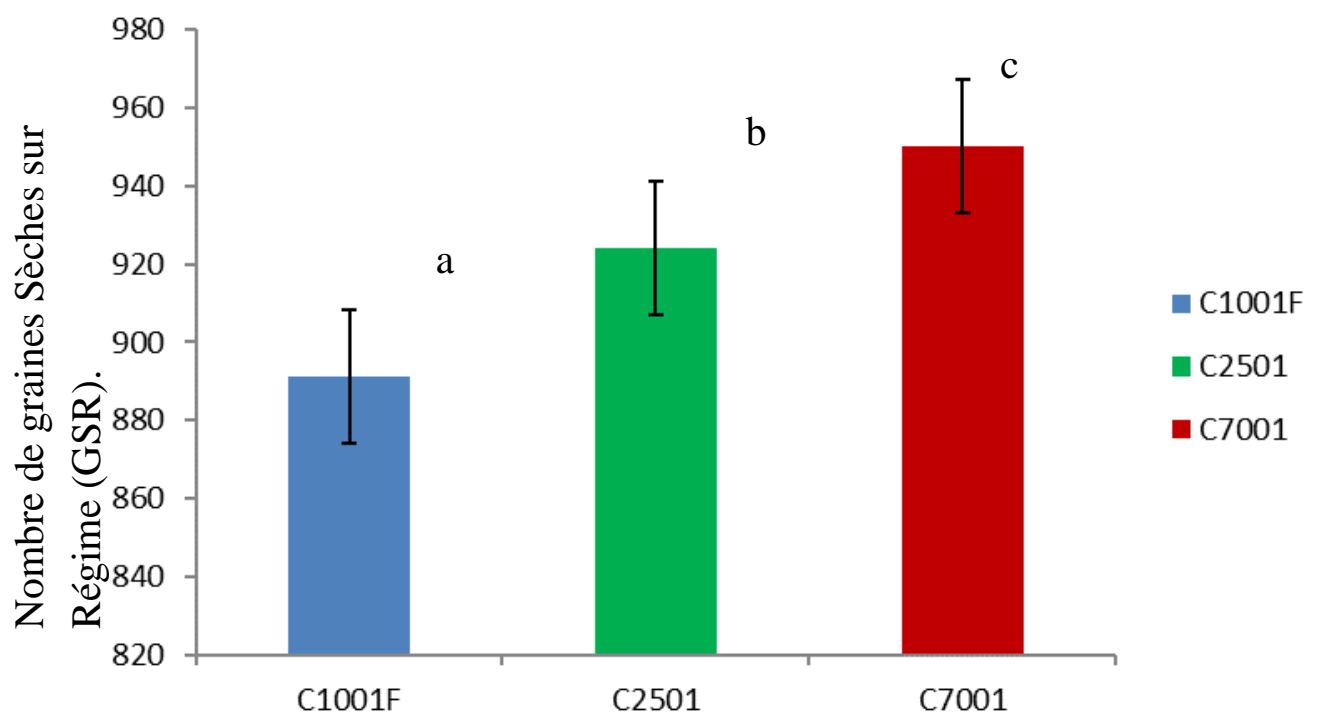

Catégories de géniteurs

Les graphiques suivis de la même lettre ne sont pas statistiquement différents au seuil de $\alpha=0,05$ par le test de KnewmanKeuls.

Figure 3: Comparaison du nombre moyen de graines sèches sur régime des trois catégories de géniteurs Dura. 
E. K. TANO et al. / Int. J. Biol. Chem. Sci. 13(3): 1800-1816, 2019

Tableau 3: Nombre de régime produit des descendances des deux systèmes de reproduction de géniteurs.

\begin{tabular}{|c|c|c|c|c|c|c|c|}
\hline Catégorie & $\begin{array}{c}\text { Couples de } \\
\text { descendance }\end{array}$ & Croisements & $\begin{array}{c}\text { Système de } \\
\text { reproduction }\end{array}$ & Descendances & $\begin{array}{c}\text { Nombre de } \\
\text { géniteurs }\end{array}$ & NR & CV $(\%)$ \\
\hline \multirow{8}{*}{$\mathrm{C} 1001 \mathrm{~F}$} & \multirow{2}{*}{ Couple 1) } & (LM2509D) AFAF & AFAF & LM19175 & 71 & $3,03 \pm 0,21^{\mathrm{a}}$ & 52,47 \\
\hline & & (LM2509D x LM2523D) AF & AFSIB & LM18503 & 22 & $3,25 \pm 0,41^{\mathrm{b}}$ & 55,07 \\
\hline & \multirow{2}{*}{ Couple 2) } & (LM3394D) AFAF & AFAF & LM17114 & 61 & $3,15 \pm 0,24^{\mathrm{a}}$ & 47,61 \\
\hline & & (LM3394D x LM2519D) AF & AFSIB & LM18783 & 66 & $4,00 \pm 0,21^{\mathrm{b}}$ & 43,00 \\
\hline & \multirow{2}{*}{ Couple 3) } & (LM2531D) AFAF & AFAF & LM19016 & 69 & $3,59 \pm 0,25^{\mathrm{a}}$ & 52,36 \\
\hline & & (LM2515D x LM2531D) AF & AFSIB & LM18805 & 100 & $3,60 \pm 0,20^{\mathrm{a}}$ & 51,66 \\
\hline & \multirow{2}{*}{ Couple 4) } & (LM3005D) AFAF & AFAF & LM19121 & 79 & $2,07 \pm 0,18^{\mathrm{a}}$ & 58,45 \\
\hline & & (LM3394D x LM3005D) AF & AFSIB & LM18801 & 85 & $3,31 \pm 0,20^{\mathrm{b}}$ & 54,68 \\
\hline \multirow{2}{*}{\multicolumn{2}{|c|}{$\mathrm{C} 1001 \mathrm{~F}$}} & $\overline{4}$ & $\overline{\mathrm{AFAF}}$ & 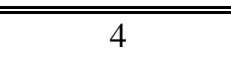 & 280 & $3,00 \pm 0,12^{\mathrm{a}}$ & 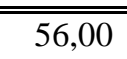 \\
\hline & & 4 & AFSIB & 4 & 273 & $3,58 \pm 0,11^{\mathrm{b}}$ & 50,83 \\
\hline \multirow{6}{*}{$\mathrm{C} 2501$} & \multirow{2}{*}{ Couple 1) } & (LM3047D) AFAF & AFAF & LM19622 & 48 & $5,46 \pm 0,34^{\mathrm{a}}$ & 39,92 \\
\hline & & (LM3047D x LM3604D) AF & AFSIB & LM19614 & 24 & $4,43 \pm 0,44^{\mathrm{b}}$ & 47,62 \\
\hline & \multirow{2}{*}{ Couple 2) } & (LM3053D) AFAF & AFAF & LM20258 & 92 & $3,43 \pm 0,20^{\mathrm{a}}$ & 55,39 \\
\hline & & (LM3053D x LM3257D) AF & AFSIB & LM21189 & 25 & $3,77 \pm 0,40^{\mathrm{a}}$ & 51,72 \\
\hline & \multirow{2}{*}{ Couple 3) } & (LM3038D) AFAF & AFAF & LM19892 & 43 & $3,58 \pm 0,38^{\mathrm{a}}$ & 69,27 \\
\hline & & (LM3038D x LM3043D) AF & AFSIB & LM19820 & 54 & $4,04 \pm 0,22^{b}$ & 47,77 \\
\hline \multirow{2}{*}{\multicolumn{2}{|c|}{$\mathrm{C} 2501$}} & 3 & AFAF & 3 & 183 & $3,95 \pm 0,17^{\mathrm{a}}$ & 54,86 \\
\hline & & 3 & AFSIB & 3 & 103 & $4,07 \pm 0,18^{b}$ & 49,03 \\
\hline \multirow{2}{*}{ C7001 } & \multirow{2}{*}{ Couple 1) } & (LM5644D) AFAF & AFAF & LM19640 & 40 & $=2,04 \pm 0,22^{\mathrm{a}}$ & 60,29 \\
\hline & & (LM5570D x LM5644D) AF & AFSIB & LM19947 & 47 & $3,52 \pm 0,30^{b}$ & 56,53 \\
\hline
\end{tabular}


E. K. TANO et al. / Int. J. Biol. Chem. Sci. 13(3): 1800-1816, 2019

\begin{tabular}{|c|c|c|c|c|c|c|}
\hline \multirow[b]{2}{*}{ Couple 2) } & (LM5373D) AFAF & AFAF & LM19759 & 30 & $3,27 \pm 0,47^{\mathrm{a}}$ & 66,36 \\
\hline & (LM5357D x LM5373D) AF & AFSIB & LM19955 & 96 & $3,53 \pm 0,25^{\mathrm{b}}$ & 64,58 \\
\hline \multirow{2}{*}{ Couple 3) } & (LM5373D) AFAF & AFAF & LM19759 & 30 & $3,27 \pm 0,47^{\mathrm{a}}$ & 64,58 \\
\hline & (LM5373D x LM5570D) AF & AFSIB & LM20983 & 107 & $4,21 \pm 0,22^{\mathrm{b}}$ & 54,63 \\
\hline \multirow{2}{*}{ C7001 } & 3 & AFAF & 3 & 100 & $2,86 \pm 0,24^{\mathrm{a}}$ & 63,74 \\
\hline & 3 & AFSIB & 3 & 250 & $3,75 \pm 0,15^{\mathrm{b}}$ & 58,58 \\
\hline
\end{tabular}

Les moyennes suivies de la même lettre dans une même colonne ne sont pas statistiquement différentes au seuil de $\alpha=0,05$ par le test $t$ de Student.

AFAF : Double autofécondation, AFSIB : Autofécondation suivie de recombinaison, NR : Nombre de régimes, Cv : Coefficient de variation.

Tableau 4 : Poids moyen de régime produit des descendances des deux systèmes de reproduction de géniteurs.

\begin{tabular}{|c|c|c|c|c|c|c|c|}
\hline Catégorie & $\begin{array}{c}\text { Couple de } \\
\text { descendance }\end{array}$ & Croisements & $\begin{array}{c}\text { Système de } \\
\text { reproduction }\end{array}$ & Descendances & $\begin{array}{l}\text { Nombre de } \\
\text { géniteurs }\end{array}$ & PMR & CV $(\%)$ \\
\hline \multirow{8}{*}{$\mathrm{C} 1001 \mathrm{~F}$} & \multirow{2}{*}{ Couple 1) } & (LM2509D) AFAF & AFAF & LM19175 & 71 & $17,36 \pm 0,61^{\mathrm{a}}$ & 25,23 \\
\hline & & (LM2509D x LM2523D) AF & AFSIB & LM18503 & 22 & $20,78 \pm 0,80^{\mathrm{b}}$ & 16,50 \\
\hline & \multirow{2}{*}{ Couple 2) } & (LM3394D) AFAF & AFAF & LM17114 & 61 & $15,91 \pm 0,56^{\mathrm{a}}$ & 22,37 \\
\hline & & (LM3394D x LM2519D) AF & AFSIB & LM18783 & 66 & $18,96 \pm 0,43^{\mathrm{b}}$ & 17,98 \\
\hline & \multirow{2}{*}{ Couple 3) } & $($ LM2531D $)$ AFAF & AFAF & LM19016 & 69 & $14,91 \pm 0,48^{\mathrm{a}}$ & 23,74 \\
\hline & & (LM2515D x LM2531D) AF & AFSIB & LM18805 & 100 & $19,06 \pm 0,43^{\mathrm{b}}$ & 20,82 \\
\hline & \multirow{2}{*}{ Couple 4) } & $($ LM3005D) AFAF & AFAF & LM19121 & 79 & $15,53 \pm 0,49^{\mathrm{a}}$ & 20,99 \\
\hline & & (LM3394D x LM3005D) AF & AFSIB & LM18801 & 85 & $21,35 \pm 0,49^{b}$ & 19,67 \\
\hline \multirow{2}{*}{\multicolumn{2}{|c|}{$\mathrm{C} 1001 \mathrm{~F}$}} & 4 & 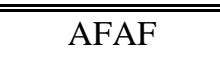 & 4 & 280 & $15,92 \pm 0,29^{\mathrm{a}}$ & 23,08 \\
\hline & & 4 & AFSIB & 4 & 273 & $20,03 \pm 0,27^{\mathrm{b}}$ & 18,74 \\
\hline \multirow{2}{*}{$\mathrm{C} 2501$} & \multirow{2}{*}{ Couple 1) } & (LM3047D) AFAF & AFAF & LM19622 & 48 & $12,01 \pm 0,43^{\mathrm{a}}$ & 24,89 \\
\hline & & (LM3047D x LM3604D) AF & AFSIB & LM19614 & 24 & $16,23 \pm 0,83^{b}$ & 25,81 \\
\hline
\end{tabular}


E. K. TANO et al. / Int. J. Biol. Chem. Sci. 13(3): 1800-1816, 2019

\begin{tabular}{|c|c|c|c|c|c|c|c|}
\hline & \multirow{2}{*}{ Couple 2) } & (LM3053D) AFAF & AFAF & LM20258 & 92 & $14,57 \pm 0,44^{\mathrm{a}}$ & 28,27 \\
\hline & & (LM3053D x LM3257D) AF & AFSIB & LM21189 & 25 & $18,99 \pm 0,77^{\mathrm{b}}$ & 19,16 \\
\hline & \multirow{2}{*}{ Couple 3) } & (LM3038D) AFAF & AFAF & LM19892 & 43 & $16,06 \pm 0,65^{\mathrm{a}}$ & 26,08 \\
\hline & & (LM3038D x LM3043D) AF & AFSIB & LM19820 & 54 & $20,06 \pm 0,55^{\mathrm{b}}$ & 23,33 \\
\hline & \multirow{2}{*}{$\mathrm{C} 2501$} & 3 & AFAF & 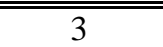 & 183 & $=14,21 \pm 0,32^{\mathrm{a}}$ & 26,41 \\
\hline & & 3 & AFSIB & 3 & 103 & $18,42 \pm 0,44^{\mathrm{b}}$ & 22,76 \\
\hline \multirow{6}{*}{ C7001 } & \multirow{2}{*}{ Couple 1) } & (LM5644D) AFAF & AFAF & LM19640 & 40 & $17,49 \pm 0,53^{\mathrm{a}}$ & 31,10 \\
\hline & & (LM5570D x LM5644D) AF & AFSIB & LM19947 & 47 & $21,38 \pm 0,79^{b}$ & 24,04 \\
\hline & \multirow{2}{*}{ Couple 2) } & (LM5373D) AFAF & AFAF & LM19759 & 30 & $19,59 \pm 0,47^{\mathrm{a}}$ & 24,70 \\
\hline & & (LM5357D x LM5373D) AF & AFSIB & LM19955 & 96 & $21,65 \pm 0,62^{\mathrm{b}}$ & 24,84 \\
\hline & \multirow{2}{*}{ Couple 3) } & (LM5373D) AFAF & AFAF & LM19759 & 30 & $19,59 \pm 1,10^{\mathrm{a}}$ & 24,70 \\
\hline & & (LM5373D x LM5570D) AF & AFSIB & LM20983 & 107 & $19,95 \pm 0,92^{\mathrm{a}}$ & 24,76 \\
\hline \multirow{2}{*}{\multicolumn{2}{|c|}{ C7001 }} & 3 & AFAF & 3 & 100 & $18,89 \pm 0,83^{\mathrm{a}}$ & 26,83 \\
\hline & & 3 & AFSIB & 3 & 250 & $20,99 \pm 0,32^{\mathrm{b}}$ & 24,54 \\
\hline
\end{tabular}

Les moyennes suivies de la même lettre dans une même colonne ne sont pas statistiquement différentes au seuil de $\alpha=0,05$ par le test $\mathrm{t}$ de Student.

AFAF : Double autofécondation, AFSIB : Autofécondation suivie de recombinaison, NR : Nombre de régimes, Cv : Coefficient de variation.

Tableau 5: Nombre de graines sèches sur régime produit des descendances des deux systèmes de reproduction de géniteurs.

\begin{tabular}{|c|c|c|c|c|c|c|c|}
\hline Catégorie & $\begin{array}{c}\text { Couple de } \\
\text { descendance }\end{array}$ & Croisements & $\begin{array}{c}\text { Système de } \\
\text { reproduction }\end{array}$ & Descendances & $\begin{array}{c}\text { Nombre de } \\
\text { géniteurs }\end{array}$ & GSR & $\mathrm{CV}(\%)$ \\
\hline \multirow{4}{*}{$\mathrm{C} 1001 \mathrm{~F}$} & \multirow{2}{*}{ Couple 1) } & (LM2509D) AFAF & AFAF & LM19175 & 71 & $855,03 \pm 34,18^{\mathrm{a}}$ & 29,12 \\
\hline & & (LM2509D x LM2523D) AF & AFSIB & LM18503 & 22 & $994,65 \pm 44,14^{\mathrm{b}}$ & 19,20 \\
\hline & \multirow{2}{*}{ Couple 2) } & (LM3394D) AFAF & AFAF & LM17114 & 61 & $752,69 \pm 42,96^{\mathrm{a}}$ & 34,56 \\
\hline & & (LM3394D x LM2519D) AF & AFSIB & LM18783 & 66 & $958,73 \pm 26,85^{\mathrm{b}}$ & 22,13 \\
\hline
\end{tabular}


E. K. TANO et al. / Int. J. Biol. Chem. Sci. 13(3): 1800-1816, 2019

\begin{tabular}{|c|c|c|c|c|c|c|c|}
\hline & \multirow{2}{*}{ Couple 3) } & (LM2531D) AFAF & AFAF & LM19016 & 69 & $708,55 \pm 32,24^{\mathrm{a}}$ & 33,38 \\
\hline & & (LM2515D x LM2531D) AF & AFSIB & LM18805 & 100 & $891,04 \pm 25,88^{b}$ & 26,34 \\
\hline & \multirow{2}{*}{ Couple 4) } & (LM3005D) AFAF & AFAF & LM19121 & 79 & $792,98 \pm 30,52^{\mathrm{a}}$ & 25,59 \\
\hline & & (LM3394D x LM3005D) AF & AFSIB & LM18801 & 85 & $1093,32 \pm 28,22^{b}$ & 22,26 \\
\hline \multirow{2}{*}{\multicolumn{2}{|c|}{$\mathrm{C} 1001 \mathrm{~F}$}} & 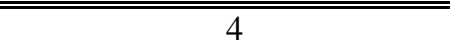 & AFAF & 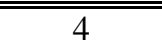 & 280 & $7 \overline{777,31 \pm 17,79^{\mathrm{a}}}$ & 30,62 \\
\hline & & 4 & AFSIB & 4 & 273 & $984,43 \pm 15,75^{\mathrm{b}}$ & 22,48 \\
\hline \multirow{6}{*}{$\mathrm{C} 2501$} & \multirow{2}{*}{ Couple 1) } & (LM3047D) AFAF & AFAF & LM19622 & 48 & $635,50 \pm 31,94^{\mathrm{a}}$ & 32,31 \\
\hline & & (LM3047D x LM3604D) AF & AFSIB & LM19614 & 24 & $755,95 \pm 53,87^{\mathrm{b}}$ & 34,10 \\
\hline & \multirow{2}{*}{ Couple 2) } & (LM3053D) AFAF & AFAF & LM20258 & 92 & $883,70 \pm 36,31^{\mathrm{a}}$ & 32,93 \\
\hline & & (LM3053D x LM3257D) AF & AFSIB & LM21189 & 25 & $1057,35 \pm 71,50^{\mathrm{b}}$ & 27,15 \\
\hline & \multirow{2}{*}{ Couple 3) } & (LM3038D) AFAF & AFAF & LM19892 & 43 & $940,20 \pm 54,86^{\mathrm{a}}$ & 37,39 \\
\hline & & (LM3038D x LM3043D) AF & AFSIB & LM19820 & 54 & $1142,90 \pm 37,56^{\mathrm{b}}$ & 27,75 \\
\hline \multirow{2}{*}{\multicolumn{2}{|c|}{$\mathrm{C} 2501$}} & 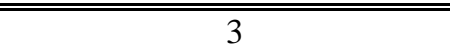 & AFAF & $\overline{3}$ & 183 & $819,8 \pm 25,93^{\mathrm{a}}$ & 34,21 \\
\hline & & 3 & AFSIB & 3 & 103 & $985,54 \pm 32,14^{\mathrm{b}}$ & 29,66 \\
\hline \multirow{6}{*}{ C7001 } & \multirow{2}{*}{ Couple 1) } & (LM5644D) AFAF & AFAF & LM19640 & 40 & $984,49 \pm 68,67^{\mathrm{a}}$ & 33,56 \\
\hline & & (LM5570D x LM5644D) AF & AFSIB & LM19947 & 47 & $994,76 \pm 50,39^{\mathrm{b}}$ & 30,34 \\
\hline & \multirow{2}{*}{ Couple 2) } & (LM5373D) AFAF & AFAF & LM19759 & 30 & $992,97 \pm 79,91^{\mathrm{a}}$ & 34,08 \\
\hline & & (LM5357D x LM5373D) AF & AFSIB & LM19955 & 96 & $1061,89 \pm 42,85^{\mathrm{b}}$ & 32,67 \\
\hline & \multirow{2}{*}{ Couple 3) } & (LM5373D) AFAF & AFAF & LM19759 & 30 & $992,97 \pm 79,91^{\mathrm{a}}$ & 34,08 \\
\hline & & (LM5373D x LM5570D) AF & AFSIB & LM20983 & 107 & $1059,16 \pm 32,96^{\mathrm{b}}$ & 29,73 \\
\hline \multirow{2}{*}{\multicolumn{2}{|c|}{ C7001 }} & 3 & AFAF & 3 & 100 & $990,14 \pm 51,94^{\mathrm{a}}$ & 33,90 \\
\hline & & 3 & AFSIB & 3 & 250 & $1038,60 \pm 24,96^{\mathrm{b}}$ & 30,91 \\
\hline
\end{tabular}

Les moyennes suivies de la même lettre dans une même colonne ne sont pas statistiquement différentes au seuil de $\alpha=0,05$ par le test t de Student.

AFAF : Double autofécondation, AFSIB : Autofécondation suivie de recombinaison, NR : Nombre de régimes, Cv : Coefficient de variation. 
Tableau 6 : Composantes de la production de régimes et variabilité des géniteurs des deux systèmes de la reproduction.

\begin{tabular}{ccccccc}
\hline $\begin{array}{c}\text { Système de la } \\
\text { reproduction } \\
\text { des géniteurs }\end{array}$ & NR & $\mathbf{C v}(\%)$ & PMR (kg) & Cv (\%) & NGSR & Cv (\%) \\
\hline AFAF & $3,95 \pm 0,09^{\mathrm{a}}$ & 56,72 & $16,24 \pm 0,22^{\mathrm{a}}$ & 27,77 & $831,35 \pm 14,97^{\mathrm{a}}$ & 35,34 \\
AFSIB & $4,07 \pm 0,08^{\mathrm{b}}$ & 48,64 & $19,31 \pm 0,20^{\mathrm{b}}$ & 25,81 & $980,92 \pm 13,02^{\mathrm{b}}$ & 30,87 \\
\hline
\end{tabular}

Les moyennes suivies de la même lettre dans une même colonne ne sont pas statistiquement différentes au seuil de $\alpha=0,05$ par le test $\mathrm{t}$ de Student

NR : Nombre de régimes ; PMR : Poids moyen du régime $(\mathrm{kg})$; NGSR : Nombre de graines sèches sur régime, Cv :

Coefficient de variation.

\section{DISCUSSION}

Les résultats présentés montrent qu'il existe une différence significative entre les variables liées à la composante de la production de régimes (nombre et poids moyen de régime et le nombre de graines sèches sur régime) des catégories de semences. Les géniteurs de la catégorie C2501 avec comme pour croisement d'origine les géniteurs DA3D x DA5D, a produit un grand nombre de régimes que les catégories C7001 et $\mathrm{C} 1001 \mathrm{~F}$ qui ont pour origine respectivement les géniteurs DA115D x DA3D et DA115D AF. Cette catégorie est caractérisée par un grand nombre de petits régimes et peu de graines sèches sur régime. Cela suppose que les gènes qui gouvernent ces caractères diffèrent d'une origine à une autre et que certaines origines de palmiers sont plus productives que d'autres. Selon Meunier et al., (1989), la production de régimes est corrélée positivement au taux de féminité donc au sexratio. Par ailleurs, l'augmentation du nombre de régimes et par conséquent l'augmentation du sex-ratio, constitue l'un des facteurs majeurs de l'accroissement de la productivité du palmier à huile. Pour Bredas et Scuvie (1960), le nombre de régime émis des palmiers dépend pour une grande part de la différenciation sexuelle influencé par le génotype mais également des conditions climatiques notamment la pluviométrie. Selon eux, le poids moyen de régime serait la résultante du génotype mais surtout des conditions écologiques qui règnent au cours de la période comprise entre la fécondation et la maturité des régimes. Une importante dispersion a été également observée au niveau de toutes les composantes de production de régimes au sein de chaque catégorie. Elle pourrait s'expliquer par l'existence d'individus génétiquement différents au sein de la même catégorie comme l'ont montré corley (1976) et Rajanaidu et al. (1981) au cours de leurs travaux. En effet, le palmier à huile est une plante à reproduction sexuée où coexistent différents génotypes au sein d'une même descendance selon l'état homozygote ou hétérozygote des gènes impliqués dans un caractère ainsi que leur nombre et les relations qui les lient.

Comme toutes les plantes allogames, le palmier à huile est sensible à la dépression de consanguinité, qui se manifeste dans les autofécondations, par une baisse de production de $50 \%$ par rapport aux hybrides (Gascon et al., 1969). Il a été constaté de façon récurrente qu'une baisse de la moyenne $\mathrm{du}$ caractère mesuré était toujours accompagnée d'une augmentation du coefficient de consanguinité moyen. Les résultats présentés dans cet article mettent en évidence l'effet dépressif de la consanguinité provoqué par les générations successives d'autofécondation pour la reproduction des géniteurs sélectionnés dans les champs semenciers. Le nombre et poids moyen de régime et le nombre de graines sèches sur 
régime ont été plus faibles au niveau des descendants issus d'autofécondation (AFAF) par rapport aux descendants issus de recombinaison (AFSIB). Le nombre de régime émis des géniteurs est toujours corrélé négativement à leur poids moyen. Cependant, les résultats ont montré que les palmiers issus de croisement de type AFAF ont donné en même temps de petit nombre de petits régimes et vice versa. Le faible poids moyen des régimes serait lié au faible nombre de graines sèches sur régime fournis par ces arbres qui, en situation de consanguinité, ont les stigmates des inflorescences femelles qui tendent à former de nombreux fruits parthénocarpiques. Ce constat, confirmé par nos études, a déjà été évoqué par des auteurs comme Gascon et al. (1969). La baisse de la production chez les géniteurs AFAF pourrait s'expliquer par la présence de gènes défavorables chez ces individus qui sont à la base de la réduction de leur vigueur. Ceci a été constaté et vérifié au Congo (SPARNAAIJ) et au Nigeria (NIFOR). En Côte-d'Ivoire, la consanguinité peut diminuer la production de régimes de près de $50 \%$ dans les autofécondations et de $25 \%$ dans les backcross avec un parent Deli (Gascon et al. 1969).En Malaisie, Hardon (1970) a mis en évidence une corrélation négative significative entre la production et des coefficients de consanguinité variant de 0,1 à 0,5 . D'ailleurs les travaux de Gascon et De Berchoux (1964), sur la comparaison de la production de différentes populations (Deli, La Mé, Yangambi) à celle d'hybrides de ces populations (Deli x La Mé, Deli x Yangambi), a mis en évidence la supériorité des croisements réalisés entre des origines génétiques différentes montrant l'existence d'un effet d'hétérosis chez le palmier à huile par rapport aux croisements intra origines. Selon ces auteurs, la consanguinité due à l'autofécondation provoque l'existence de gynandromorphes chez les plantes et réduit la production de régimes. La dépression de la consanguinité est due non seulement à la fixation à l'état homozygote d'allèles défavorables au niveau des systèmes polygéniques mais aussi à la réduction de l'hétérozygotie entrainant une rupture des interactions alléliques et en conséquence une diminution de l'hétérosis provoquant une baisse de la productivité. Gallais (1990), a aussi montré une baisse du rendement chez la luzerne due à des autofécondations. Cela a été constaté chez l'amandier, plante de la famille des Rosacées, où les effets de consanguinité se sont manifestés par une augmentation de la période juvénile (Noiton et al., 1996) ou par une très faible productivité (Grasselly et al., 1981). Aussi, les coefficients de variabilité enregistrés ont été plus élevés chez les géniteurs issues de reproduction de type AFAF que chez ceux obtenus à partir de reproduction de type AFSIB. Cette importante dispersion s'expliquerait par la dérive génétique due aux effets néfastes de la consanguinité qui a pu créer des mutations dans le matériel nucléaire ou cytoplasmique chez les géniteurs issus des séries d'autofécondation en augmentant la variabilité en leur sein.

Comme chez les végétaux, de nombreuses études ont tenté de mettre en évidence la relation entre la consanguinité et la baisse de performance à la fois pour les qualités d'élevage que pour les caractères de production. Chez les pigeons, l'effet du degré de consanguinité se manifeste à partir de la deuxième semaine d'âge et entrâne une dépression de la consanguinité sur le poids. Selon Soulaymani et al., (1997), la consanguinité chez les pigeons avantage les mâles par rapport aux femelles et entraine une perte de poids chez les pigeonneaux. Ces auteurs ont effectué des comparaisons multiples sur les moyennes des caractères et ont découvert à l'intérieur de chaque groupe un avantage des individus non consanguins sur les autres. L'étude des corrélations a aussi montré des relations inversement proportionnelles entre le poids des pigeonneaux, d'une part, et leurs sexes et /ou leurs degrés de consanguinité. Les résultats observés concordent avec les travaux de Dobzhansky et Spassky (1963), Stone et al., (1963), Malogolowkin et al. (1964) sur la 
drosophile. L'étude des régressions a permis de quantifier le degré de liaison entre le poids et la consanguinité. Une étude réalisée en Turquie a révélé que la proportion d'avortements est deux fois supérieure chez les couples du premier degré par rapport aux non consanguins (Hussain, 1998). La grande majorité des données dans la littérature montre que la consanguinité tend à augmenter la morbidité et la mortalité. D'après (Biémont et al., 1974), les effets néfastes des croisements consanguins sont dus au fait que les homozygotes sont moins bien adaptés que les hétérozygotes et que ce sont les populations où les individus qui ont un degré d'hétérozygotie élevé qui présentent la valeur sélective optimale. Pour ces auteurs, la consanguinité diminuerait le pool d'enzymes contrôlant les réactions du métabolisme et par conséquent perturberait le système de régulation d'homéostasie physiologique des individus consanguins. Selon (Solignac et al., 1995), les individus non consanguins ont une plus grande variabilité biochimique qui leur permet de mieux répondre aux variations du milieu. On parle alors de pouvoir homéostatique réduit chez les individus consanguins. $\mathrm{Ce}$ phénomène appelé "dépression de consanguinité" se manifeste de façon très nette sur les caractères liés à la fitness des organismes, réduisant ainsi fortement la fertilité, la fécondité et la longévité. D'après (Biemont, 1974), la consanguinité provoque une perturbation des interactions entre gamètes, ce qui explique la sensibilité de ces organes sexuels à la consanguinité. Le taux d'avortements spontanés est d'autant plus élevé dans les familles où la consanguinité se perpétue depuis plusieurs générations à cause de l'effet d'homozygotie croissante sur le développement du foetus (Hussain, 1998 ; Bener et Alali., 2006).

Mes résultats ne concordent cependant pas avec ceux de Hardon et Chai (1971) qui ont montré dans leur étude que certains caractères chez le palmier ne sembleraient pas être affectés par la consanguinité. Il s'agit des caractères présentant une héritabilité élevée qui sont utilisés comme marqueurs au cours de la sélection des parents (Jacquemard et al., 1981).

\section{Conclusion}

Cette étude a consisté à évaluer deux systèmes de reproduction de géniteurs utilisés en production de semences à la station CNRA de La Mé (Côte d'Ivoire) chez trois catégories de géniteurs C1001F, C7001 et C250, afin de proposer une meilleure stratégie de création de futurs champs semenciers de palmier à huile. Pour cela les composantes de production de quelques descendances issues d'autofécondation et de recombinaison de ces trois (3) catégories de semences ont été mesurées et comparées. La mesure et la comparaison de ces différentes variables que sont le nombre et poids moyen de régime et le nombre de graines sèches sur régime a montré que parmi les trois catégories, la catégorie C2501 a produit un grand nombre de petit régime. Quant à la catégorie C7001, elle a fourni de gros régimes, avec un nombre élevé de graines sèches sur régime. La catégorie C1001F a donné petit nombre de petit régime et un petit nombre de graines sèches sur régime. La comparaison des deux systèmes de reproduction des géniteurs a révélé que le nombre et poids moyen de régime et le nombre de graines sèches sur régime ont été plus faibles chez les géniteurs autofécondés issus de croisement de type (A1 x A1) AF (double autofécondation) que chez les géniteurs issus de recombinaisons parentales de type (A1 $x$ A2) suivie d'une autofécondation $(\mathrm{AF})$.

\section{CONFLIT D'INTERETS}

Les auteurs déclarent qu'il n'y a aucun conflit d'intérêts.

\section{CONTRIBUTIONS DES AUTEURS}

Tous les auteurs ont contribué à la réalisation de ce travail, et EKT était l'investigateur principal. Tous ont contribué à la correction du manuscrit. 


\section{REFERENCES}

Beirnaert A. 1935. Germination des graines d'Elaeis. Institut National Agronomique du Congo Belge, 4 : 3-39.

Bernard G, Maliingraux C. 1965. Production de semences sélectionnées de palmiers a huiles à I.R.H.O. Oléagineux, 20(5): 297-305.

Bener A, Alali KA. 2006. Consanguineous marriage in a newly developed country: the Qatari population. J. Biosoc Sci, 38: 239-246.

Biémont C, Bouffette AR, Bouffette J. 1974. Théorie chromosomique de l'inbreeding: Modèle probabiliste. Bulletin of Mathematical Biology, 36 : 417 - 434.

Bouroche JM, Saporta G. 1980. L'analyse des données. Collection Que Sais-Je, PUF. Dunod. XV, 62 P.

Bredas J, Scuvie L. 1960. Aperçu des influences climatiques sur les cycles de production du palmier à huile, Oléagineux, 15(4) : 211 - 222.

Cheng HT. 2010. Les principaux enjeux du développement durable dans le secteur de l'huile de palme. Un document argumenté pour les consultations des différents acteurs. (Commissionné par la Banque Mondiale). p 1- 52.

Corley RHV, Tinker PB. 2016. The Oil Palm. (5e édn). John Wiley \& Sons, Ltd.: Oxford, United Kingdom.

Corley RHV. 1976. Inflorescences l'avortement et de différentiation sexuelle, Ed, progrès de la science des cultures. Elsewer, Amsterdan Pays Bas. 1: 23-36.

Dagnelie P. 2012. Principes d'Expérimentation: Planification des Expériences et Analyses de leurs Résultats. Les Presses Agronomiques de Gembloux, passage des déportés, 2 : B5030 Gembloux, Belgique ; 413 p.

Dobzhansky Th, et Spassky B, 1963. Genetics ofnatural populations XXXIV. Adaptive norm, genetic load, and genetic elite in Drosophila pseudoobscura. Genetics, 48: 1467-1485.

Durand-gasselin T, de Franqueville H, Breton F, Amblard P, Jacquemard J-C, Syaputra
I. 2011. Breeding for sustainable palm oil. Int. Semin. Breed. Sustain. Oil Palm. 178-193.

Durand-gasselin T, Blangy L, Picasso C, Breton F, Amblard P, Cochard B. 2010. Sélection du palmier à huile pour une huile de palme durable et responsabilité sociale. OCL, 17: 385-392.

Gallais A. 1990. Théorie de la Sélection en Amélioration des Plantes. Ed. Masson: Paris (France) ; 588 p.

Gascon JP, Jacquemard JC, Houssou M, Boutin D, Chaillard H, Kamga F. 1981. La production de semences sélectionnées de palmier à huile (Elaeis guineensis Jacq.). Oléagineux, 36(10) : 475-486.

Gascon JP, De Berchoux C. 1964. Caractéristiques de la production d'Elaeis guineensis Jacq. de diverses origines et de leurs croisements: Application à la sélection du palmier à huile. Oléagineux, 19 (2): 75-84.

Grasselly CH, Crossa-Raynaud P, Olivier G, Gall H. 1981. Transmission du caractère d'autocompatibilité chez l'amandier, Colloque Grempa, Options Méditerranéennes, Ciheam / Iamz 81/I. pp. 71-75.

Hardon JJ. 1970. Inbreeding in populations of the oil palm and its effects on selection. Oléagineux, 25: 85-90.

Hardon JJ, Ooi SC. 1971. To what extent should inbreeding be avoided in oil palm seed production. Chemara Research Station, Malaysia. Communication (agronomie), restricted distribution, 9: $11 \mathrm{p}$.

Henry P. 1955. Morphologie de la feuille d'essai au cours de sa croissance. Rev. Gén. Bot., pp. 319 - 323.

Houssou M. 1985. Amélioration du palmier à huile en zone peu humide. Résultats récents obtenus au Bénin. Thèse Doct. Ing, Université PARIS-SUD ORSAY, $119 \mathrm{p}$.

Hussain R. 1998. The role of consanguinity and inbreeding as a determinant of spontaneous abortion in Karachi, Pakistan. Annals of Human Genetics, 62(2): 147-157. 
Jacquemard JC, Meunier J, Bonnot F. 1981. Etude génétique de la reproduction d'un croisement chez le palmier à huile Elaeis guineensis: Application à la production de semences sélectionnées et à l'amélioration. Oléagineux, 36(7): 343349.

Malogolowkin C, Levene H, Dobzhansky NP, Simmons S. 1964. Inbreeding and mutational and balanced loads in natural populations of Drosophila willistoni. Genetics, 50: 1299-1311.

Meunier J, Potier F, Amblard P, Tailliez B. 1989. Relations entre la production d'huile et le nombre de régimes chez le palmier à huile (Elaeis guineensis Jacq.): conséquences pour la pollinisation dans les jeunes plantations. Oléagineux, 44(6): 269-279.

Meunier J, Gascon JP. 1972. Le schéma général d'amélioration du palmier à huile à l'I.R.H.O. Oléagineux, 27 (1): 1-12.

Noiton DAM, Alsprach P. 1996. Founding clones, inbreeding, coancestry, and status number of modern apple cultivars, J. Am. Soc. Hortic. Sci., 121 (5) : 773-782.

Osseni, 2009. La filière palmier à huile, la filière du progrès. Bulletin d'information $d u$ Fond Interprofessionnel pour la Recherche et le Conseil Agricole, 5: $54 \mathrm{p}$.

Rajanaidu N, Lawrence MJ, Oil SC. 1981. Variation in nigerian oil palm germplasm and ils relevance to oil palm breading in the oil palm. In Agriculture in the Eighties, Pushparajah E, Chew PS (eds). lsp Kuala Lumpur.
Rival A, Levang P. 2014. Palms of Controversies: Oil Palm and Development Challenges. CIFOR: Bogor, Indonesia.

Solignac M, Periquet G, Anxolabehere D, Petit C. 1995. Génétique et Evolution 1: La Variation des Gènes dans les Populations. Collect. Meth., Herman, Ed des Sciences et des Arts, pp 289.

Soulaymani A, Benazzouz B, Mokhtari A. 1997. Incidence de la consanguinité sur la viabilité des pigeonneaux. Reu. Fac. Sci. Marrakech, 9: 27-33.

Stone WS, Wilson FD et Gerstenberg VL. 1963. Genetic studies of natural populations of drosophila, a large dominant population. Genetics, 48: 10891106.

Tano EK, Allou D, Alla O, Konan KE, N'Guetta ASP. 2017. Evaluation of the height of some genitors of seed field Deli Dura of Oil Palm (Eleais guineensis Jacq) planted in 1998 and 1999 at $\mathrm{La} \mathrm{Me}$ Station. Agricultural Science Research Journal, 7(10): 318 - 323 .

USDA. 2017. Oil seeds: world market and trade. Foreign Agricultural Service, Circular Series March 2017. http//apps.fas.usda.gov/psdonline/circula rs/production.pdf (consulté le 3.16.17). 